\title{
Parenting dimensions in mothers and fathers of children with Autism Spectrum Disorders
}

\author{
Yagmur Ozturk ${ }^{\mathrm{a}, *}$, Samantha Riccadonna ${ }^{\mathrm{b}}$, Paola Venuti ${ }^{\mathrm{a}}$ \\ ${ }^{a}$ Department of Psychology and Cognitive Science, University of Trento, Rovereto, Italy \\ ${ }^{\mathrm{b}}$ Department of Computational Biology, Research and Innovation Centre, Fondazione Edmund Mach (FEM), San Michele all'Adige, Italy
}

\section{A R T I C L E I N F O}

\section{Article history:}

Received 4 October 2013

Received in revised form 27 June 2014

Accepted 4 July 2014

Available online 26 July 2014

\section{Keywords:}

Autism Spectrum Disorder (ASD)

Mothers and fathers

Parental stress

Parental attitude

Parental mental health

\begin{abstract}
A B S T R A C T
Rearing a child with Autism Spectrum Disorder (ASD) is a unique challenge for both parents. Previous studies addressed how mothers are affected by the challenges of raising a child with ASD, mostly in terms of stress pattern. In this study, we focused on comparisons between mothers and fathers of children with ASD in parental stress, attitude and mental health. We examined 99 parents of children with ASD using the Parenting Stress Index-Short Form, the Parental Style Questionnaire, the Self-Perceptions of the Parental Role and the Symptom Checklist-90-Revised. The results revealed the gender differences in the parental attitude and mental health. Mothers reported that they engaged in more social behaviors with their children than fathers. In addition mothers reported higher level of depression than fathers. No difference among parents emerged in the Parenting Stress Index-Short Form. The results of a multiple regression analysis revealed that parenting distress is associated with depression, balance of parents' diverse roles in their life and dysfunctional interaction between parents and children. These findings highlight both similarities and differences between mothers and fathers of children with ASD and the existence of a relationship between parental stress, mental health and attitude. Results suggest the importance of developing specific intervention programs which incorporate these fundamental parenting domains.
\end{abstract}

() 2014 Elsevier Ltd. All rights reserved.

\section{Introduction}

Autism Spectrum Disorder (ASD) is a complex neurodevelopmental disorder characterized by difficulties in social interaction and communication as well as the presence of restricted, repetitive patterns of behavior, interests, or activities (American Psychiatric Association, 2013). These difficulties do not only affect the diagnosed people throughout their life, but also their parents who play salient and influential roles in child development (Karst \& Van Hecke, 2012). Parenting an infant constitutes the initial and all-encompassing ecology of infant development (Bornstein, 2002) and it is a process which constitutes a key foundational component in the child's life. In addition, the birth of any child with special needs presents significant difficulties to the parents. Taking into account the characteristics of ASD, rearing a child with those developmental difficulties is an important and unique challenge for both mothers and fathers.

\footnotetext{
* Corresponding author at: Department of Psychology and Cognitive Science, University of Trento, Via Matteo del Ben 5B, 38086 Rovereto, Italy. Tel.: +390464808115.

E-mail address: yagmur.ozturk@unitn.it (Y. Ozturk).
} 
In the last decade many studies have tended to focus on how parents are affected by the challenges of raising a child with ASD, particularly in terms of their stress (Baker-Ericzn, Brookman-Frazee, \& Stahmer, 2005; Davis \& Carter, 2008; Eisenhower, Baker, \& Blacher, 2005; Hastings, 2003; Hoffman, Sweeney, Hodge, Lopez-Wagner, \& Looney, 2009; Moes, Koegel, Schreibman, \& Loos, 1992; Sharpley, Bitsika, \& Efremidis, 1997; Tehee, Honan, \& Hevey, 2009). However, far too little attention has been paid to other important domains of parenting, such as parental attitude which is the way parents behave, and mental health which describes a level of psychological well-being. In addition, there is a little discussion about fathers of children with ASD, even though fathers are important as mothers in children's development (Parke, 2002). In the present study, we aimed to examine the profiles of both mothers and fathers of children with ASD addressing parenting stress, parental mental health, and parental attitude. It is crucial to take parents into account in order to gain a better and deeper understanding of family dynamics of children with ASD, and to develop strategies to supports both parents and children.

One of most frequently examined aspects of parenting is stress. In the past decade, a number of researchers have attempted to describe parenting stress (Crnic \& Low, 2002; Deater-Deckard, 2004), as a set of processes that lead to aversive psychological and physiological reactions arising from attempts to adapt to the demands and it is often experienced as negative feelings toward and about the self and the child (Deater-Deckard, 2004). A variety of interacting variables contribute over time to parents' perception of stress, such as presence of challenging situations (Crnic \& Low, 2002; Deater-Deckard, 2004). Considering the fact that ASD affects not only the world of a child, but also the parents' life, the child's disability which is a considerable challenging situation is a fundamental variable to take into account in the topic of parenting stress.

The majority of studies have focused on maternal stress, mostly comparison between mothers who are differentiated due to their children's conditions (e.g., existence of disability). Eisenhower and colleagues (2005) found that mothers of children with ASD demonstrated more parenting stress than mothers of children with Down syndrome, undifferentiated developmental delays, cerebral palsy and typical development. Similar results were obtained by Estes, Munson, Dawson, and Koehler (2009) in their comparison of mothers of children with ASD and children with developmental delay without autism. Hoffman and colleagues (2009) reported the difference also between mothers of children with ASD and mothers of typically developing children.

However, little attention has been specifically directed toward fathers (Flippin \& Crais, 2011). In 1992, Rodrigue, Morgan, and Geffken (1992) suggested that fathers adapt relatively well to the demands associated with raising a child with a developmental disability. With respect to fathers of children with ASD, fathers as well as mothers, were found to have elevated stress compared to those of typically developing children (Baker-Ericzn et al., 2005; Rao \& Beidel, 2009), however there is no general agreement in whether one of parents of children with ASD experience higher level of stress than other. Some studies reported that mothers were significantly more stressed and more involved than fathers (Moes et al., 1992; Sharpley et al., 1997; Tehee et al., 2009), other studies showed that mothers and fathers had similar stress levels (Davis \& Carter, 2008; Hastings, 2003). Similar debate stands in the researches which focused on the comparisons between mothers and fathers of children with other disabilities or without disabilities (Baker, 1994; Beckman, 1991; Deater-Deckard, 2004; Perry, Sarlo-McGarvey, \& Factor, 1992; Theule, Wiener, Tannock, \& Jenkins, 2010). It is interesting to note that, although they had similar stress levels, fathers of children with ASD were mostly distressed by the child's externalizing problems, while mothers were more affected by the child's regulatory problems (Davis \& Carter, 2008).

Although many studies of parenting a child with ASD have concentrated in parenting stress, also mental health and wellbeing of parents are an important domain of parenting. Mental health has been shown to have effects on parenting; compared to non-depressed woman, depressed mothers have been found to be more negative with their children from infancy through adolescence (Foster, Garber, \& Durlak, 2008; Jacob \& Johnson, 1997). Moreover, it has been pointed out that paternal and maternal depression were similarly associated with child adjustment problems and more impaired parentchild communication (Gottman \& Wilson, 2002; Jacob \& Johnson, 1997). In addition, well-being of adults and children alike is linked to the types and degrees of stressful circumstances that they face in their daily lives; those who experience more stressful events are more likely to suffer from depression and other problems in mental and physical health (see DeaterDeckard, 2004).

In terms of comparison between parents who are differentiated due to their children's conditions, researchers showed that parents of children with ASD demonstrated higher levels of mental health concerns than parents of typically developing children as well as children with other impairments such as intellectual disability (Gau et al., 2012; Karst \& Van Hecke, 2012; Olsson \& Hwang, 2001). However, there is no agreement in the existence of similarities or differences in the level of various psychopathologies between mothers and fathers of children with ASD. Some studies reported that mothers have higher levels of depression (Davis \& Carter, 2008; Hastings et al., 2005; Olsson \& Hwang, 2001) and anxiety (Hastings, 2003) than fathers, other studies found no differences in depression (Hastings, 2003).

Another well-known domain of parenting is parental attitude. All we know about parental attitudes toward rearing a child with ASD come from studies on parental beliefs and style. Parental belief is a perception about childrearing and an idea on which components parenting consists of. Specifically, beliefs are key aspects of parenting because they generate and organize parental behaviors and mediate the effectiveness of parenting and ultimately affect child development (Bornstein \& Lansford, 2010; Bornstein \& Venuti, 2013; Bornstein, 2002; Darling \& Steinberg, 1993; Senese, Bornstein, Haynes, Rossi, \& Venuti, 2012). In terms of the different parents' beliefs, Melson, Ladd, and Hsu (1993) found that mothers who attributed difficulty in helping their children to attain specific qualities perceived more difficulty than did those mothers who made casual attributions to maternal characteristics or behavior. Crnic and Low (2002) suggested that this might be especially salient for children who have special needs. 
In addition to parental belief, a number of studies focused on the importance of parenting style on academic achievement of children in schools highlighting (Cohen \& Rice, 1997; Fan \& Chen, 2001; McGrath \& Repetti, 2000). With respect to the gender differences, a number of reviews suggest that parenting behavior of mothers and fathers differ in several important ways, such as father involvement in infancy and childhood is quantitatively less than mother involvement (Barnard \& Solchany, 2002; Parke, 2002). Mothers spend more time with their children, and the interactions are characterized by the mother's caregiving and managerial role, whereas fathers spend more of their time with their children in play activities.

Although parenting attitude is important for the child development, surprisingly, it has not been explored from the point of parents of children with ASD. Nevertheless it is essential to understand caregivers' belief in their own ability to parent their child and in their attitudes toward child rearing. Therefore, in our study, we aim to consider the parental attitude as one of important domains of parenting.

In sum, to date, the studies have not covered parenting a child with ASD in much detail, the majority of research has been conducted with mothers and mostly in terms of maternal stress pattern. In the present study, we aimed to draw a comprehensive profile of both mothers and fathers who have children with ASD addressing all the important domains of parenting. In particular the goals of this study are: (1) to determine whether mothers and fathers differ in terms of parenting stress, parental attitude and parental health; (2) to examine the relationship between the parents' attitude toward childrearing, the mental health of both parents and their experience of stress. Guided by the literature on parenting, highlighting the differences between mothers and fathers in parenting stress and mental health (Davis \& Carter, 2008; Hastings et al., 2005; Hastings, 2003; Moes et al., 1992; Olsson \& Hwang, 2001; Rodrigue et al., 1992; Sharpley et al., 1997; Tehee et al., 2009) we hypothesized that mothers and fathers differ in terms of parenting stress, mental health and attitude. In addition, we expected an association between parenting stress, mental health and attitude.

\section{Method}

\subsection{Participants and procedure}

The participants in the present study were 99 parents (50 mothers: $M$ age $=40.67$ years, $S D=5.66$; 49 fathers: $M$ age $=44.1$ years, $S D=5.72$ ) of children with ASD and 94 of them were paired (both mother and father of a child were enrolled). The children's average age at the time of the study was 87.5 months ( $S D=40.9$ months). The diagnosis of children was confirmed through clinical judgment by an independent clinician based on the DSM-IV-TR criteria for Pervasive Developmental Disorders (PDD) as well as through the Autism Diagnostic Observation Schedule-Generic (ADOS-G; Lord, Rutter, DiLavore, \& Risi, 2003) in the Observation, Diagnosis and Education Lab at the University of Trento, Italy (27\% of the sample were autistic children with high cognitive function, $36 \%$ of the sample were autistic children with low cognitive function, $25 \%$ were pervasive developmental disorder not otherwise specified, and $12 \%$ were Asperger syndrome). One of three modules was used for the children passed the cut-off points for the Autism Spectrum Disorder. The socioeconomic status (SES) of the parents was calculated with the Four-Factor Index of Social Status (Hollingshead, 1975; Rossi, 1994) which is today the most widely adopted index of SES in psychological research (Bornstein, Haynes, O'Reilly, \& Painter, 1996; Bornstein et al., 2003; De Falco, Esposito, Venuti, \& Bornstein, 2008; Gottfried, 1985; Ribas et al., 2003; Rossi, 1994; Venuti \& Senese, 2007; Venuti, de Falco, Giusti, \& Bornstein, 2008). In the present study, our sample represented a middle status in the Italian population $(M=36.42, S D=16.5)$.

Parents were given four questionnaires: the Parenting Stress Index-Short Form (PSI/SF; Abidin, 1995), the Parental Style Questionnaire (PSQ; Bornstein, Haynes, et al., 1996; Bornstein et al., 1996), the Self-Perceptions of the Parental Role (SPPR; MacPhee \& Benson, 1986) and the Symptom Checklist-90-Revised (SCL-90-R; Derogatis \& Lazarus, 1994). Both parents completed the questionnaires on their own (taking approximately $45 \mathrm{~min}$ to do so) and returned them to the researchers.

\subsection{Measures}

\subsubsection{The Parenting Stress Index-Short Form}

(PSI/SF; Abidin, 1995) is a self-reported measure designed to evaluate parenting stress. It consists of 36 items that parents respond to on a 5 point Likert-type scale to indicate the degree to which the participants agree with each statement, ranging from 1 (Strongly Agree) to 5 (Strongly Disagree). The PSI/SF is composed of three scales: Parental Distress (PD), Difficult Child Characteristics (DC) and Parent-Child Dysfunctional Interaction (P-CDI). The PD scale consists of items relating the distress parents experience in their roles as parents as a function of personal factors that are directly related to parenting. A sample item from the PD scale is: "Since having a child I feel that I am almost never able to do things I like to do". The DC scale measures parents' perception related to some of the basic behavioral characteristics of children that make them easy or difficult to manage, for example, "My child turned out to be more of a problem than I had expected". The P-CDI scale focuses on parents' perceptions of the interaction system between the parent and child through items such as, "Most times I feel that my child does not like me and does not want to be close to me". The PSI includes a Defensive Responding scale (DF) that indicates the degree to which the parent might be attempting to give a more positive self-image, deny stress or minimize problems in their relationship with their child. The PSI also yields a Total Stress score (TS) that is the sum of PD, P-CDI and DC to provide an indication of the total level of parental stress that an individual is experiencing in his/her role as a parent. 
In general, for each scale, a score ranging between the 15th and 80th percentile is considered typical while equal or greater than the 85th percentile (the clinical cut-point) is considered as high. A score on the DF scale equal to or more than the 10th percentile indicates high levels of defensive responding. The three scales and the total scale have shown high internal consistency (Abidin, 1995; Guarino, Di Blasio, D’Alessio, Camisasca, \& Serantoni, 2007). In the present sample, alphas ranged from .84 to .92 for mothers across the three scales and the total scale. For fathers, alphas on these scales ranged from .81 to .91 .

\subsubsection{The Parental Style Questionnaire}

(PSQ; Bornstein, Haynes, et al., 1996; Bornstein et al., 1996) is a self-reported measure of parenting behavior. It consists of 16 items assessing three parenting style: Social Exchange (sensitivity, expressions of affection, and positive responsiveness to the child), Didactic/Material (providing stimulation and organizing an environment conducive to exploration), and Limit Setting (emphasizing rule keeping and mannerliness in the child). Parents were asked to rate the items on a 5-point Likerttype scale (from 1 hardly at all to 5 all the time) how frequently they actually engage in specific parenting behaviors. The PSQ scales have demonstrated good internal consistency and construct validity (Bornstein, Haynes, et al., 1996; Bornstein et al., 1996; Bornstein, Cote, \& Venuti, 2001; Venuti \& Senese, 2007). For the present study, internal consistencies ranged from .71 to .78 for mothers, and .68 to .80 for fathers.

\subsubsection{The Self-Perceptions of the Parental Role}

(SPPR; MacPhee \& Benson, 1986) is a self-reported measure containing 22 items to assess the four parental selfperceptions: Competence, Satisfaction, Investment, and Role Balance. Each item has a pair of statements that describes contrasting endpoints of the dimension to minimize socially desirable responses. The parents were asked to choose the statement that describes their best and then checks "sort of true for me" or "really true for me." Possible scores range from 1 (low perceived competence, satisfaction, investment, or role balance) to 5 (high perceived competence, satisfaction, investment, or role balance). SPPR scales have shown good internal consistency, Cronbach's alphas ranged from .72 to .80 across the scales (MacPhee \& Benson, 1986; Seybold, Fritz, \& Macphee, 1991). The good internal consistency was confirmed in the present study with Cronbach's alphas ranged from .75 to .78 for mothers and .72 to .77 for fathers.

\subsubsection{The Symptom Checklist-90-Revised}

(SCL-90-R; Derogatis \& Lazarus, 1994) is a self-reported questionnaire containing 90 items to screen for a broad range of symptoms. It consists of 9 primary symptom dimensions: Somatization (SOM), Obsessive-Compulsive (O-C), Interpersonal Sensitivity (I-S), Depression (DEP), Anxiety (ANX), Hostility (HOS), Phobic Anxiety (PHOB), Paranoid Ideation (PAR), and Psychoticism (PSY). Parents were asked to answer to on a 5 point Likert-type scale, ranging from "not at all" (0) to "extremely” (4). The scales have demonstrated good internal consistency (Derogatis \& Lazarus, 1994; Sarno, Preti, Antonio, \& Madeddu, 2011). For the present study, the internal consistency ranged from .63 to .91 for mothers, from .62 to .81 for fathers across the scales.

\subsubsection{The Autism Diagnostic Observation Schedule-Generic}

(ADOS-G; Lord et al., 2003) is a semi-structured, standardized assessment used for diagnosing individuals with ASD. It is an assessment of the child's social interaction, communication, play, and imaginative use of materials through an interaction with a trained professional. We use ADOS-G to classify the children as having autism and to examine the relationship between parenting measures and three scores of ADOS-G which are Communication score, Reciprocal Social Interaction score, Total score (a sum of the Communication and Reciprocal Social Interactions scores). Good internal consistency have been reported by Lord and colleagues (2003).

\subsection{Data analyses}

First of all, we removed nine of the 99 parents (three mothers and six fathers) from the analysis, since they had high level scores on the Defensive Responding scale of the PSI/SF. We performed preliminary analyses on the remaining 90 parents (47 mothers and 43 fathers), reporting descriptive statistics of each parenting measures after incomplete cases were removed. Moreover, we checked if there is a correlation between the parenting measures and the ADOS-G.

Then we examined all the questionnaires to determine if there are any gender differences and associations, using only paired samples (both parents of a child). We explored gender differences testing the PSI/SF, the PSQ and the SPPR scores with paired $t$-tests, after having checked normality with the Shapiro-Wilk test. For the SCL-90-R, Wilcoxon Signed-Rank test was used due to small sample size (20 mothers and 20 fathers). Moreover, correlation analysis was performed to determine the relationship strength between parents' scale scores of the PSI/SF, the PSQ, the SPPR and the SCL-90-R, using Pearson for normally distributed variables, and Spearman correlation coefficients for the remaining comparisons. We reported correlation coefficients from moderate to strong, i.e., ranging between 0.50 and 1.00 , or between -0.5 and -1.00 , repeating the analysis for all parents together and also for mothers and fathers separately.

Finally, to further explore the relationship between parenting stress and other parenting domains, we carried out a multiple regression analysis on the 90 subjects. First, linear models were built both using forward selection and backward elimination techniques using all parenting domain measures and choosing the best model on the basis of minimum BIC score 
(Schwarz, 1978). The best model was very complex (having 10 explanatory variables), thus we repeat the model building procedure to the variables more correlated with PD. Again using the minimum BIC score approach, we choose a model with 3 explanatory variables. The model was validated using a 10-fold cross validation procedure. In what follows we report and discuss only the results of the model with 3 explanatory variables since the loss of performance of this model (adjusted $R^{2}$ equal to 0.56 for the 3 variables model versus 0.76 for the 10 variables model after the 10 -fold cross validation procedure) are balanced by better interpretability of the 10 variables model.

\section{Results}

We investigated the association between our measures on 90 parents and the corresponding demographic variables: no associations emerged between parenting measures and child age, parents' age, or family SES. Moreover, we checked if there is a correlation between the Parenting Stress Index-Short Form (PSI/SF) and the ADOS-G: a statistically significant positive correlation was found between the Total Stress (TS) score of the PSI/SF and Communication domain of the ADOS-G ( $\rho=.50$, $p<.001)$. For mothers, we observed a statistically positive correlation between TS and Communication $(\rho=.53, p<.001)$; whereas, for fathers, we detected a correlation between the Difficult Child (DC) scale of the PSI/SF and Communication ( $\rho=.50, p<.001$ ). Regarding to the other measures, a statistically significant positive correlation was emerged for fathers between the Somatization scale of the SCL-90-R and Communication domain of ADOS-G $(\rho=.57, p=.003)$.

Mean scores and percentages of subjects in clinically significant range among mothers and fathers on the PSI/SF are presented in Table 1. Tables 2 and 3 show the mean scores for the PSQ the SPPR and the SCL-90-R. The data in tables are based on different sample sizes because of incomplete data.

Focusing on gender differences, we found a statistically significant result in the Social Exchange scale of the PSQ $t(31)=3.46, p=.002$ (see Fig. 1): mothers reported higher score than fathers in this scale Moreover, a statistically significant difference emerged in the Depression scale of the SCL-90-R, $T(19)=135, p=.03$ (see Fig. 2): mothers reported higher level of depression than fathers. It is worth noting that no gender differences were found in the PSI/SF and the SPPR.

The relationships among parenting measures, i.e., between the scales are displayed in Fig. 3. We found statistically significant positive correlations both within the PSI/SF and within the SPPR. In particular, for the PSI/SF, we highlight the correlation between PD and P-CDI $(\rho=.53, p<.001)$, between PD and DC $(\rho=.55, p<.001)$, and between P-CDI and DC $(\rho=.58, p<.001)$, while within the SPPR, between Role Balance and Satisfaction $(\rho=.56, p<.001)$. Moreover, we observed statistically significant positive correlations between the PSI/SF and the SCL-90-R: PD and Depression $(\rho=.56, p<.001)$, PD and Hostility $(\rho=.55, p<.001)$. Furthermore, statistically significant negative correlations between the PSI/SF and the SPPR emerged: PD and Role Balance $(\rho=-.60, p<.001)$, TS and Role Balance $(\rho=-.56, p<.001)$.

Focusing separately on mothers or fathers some differences emerged. In particular, for mothers, two over three correlations were confirmed within the PSI/SF: PD and P-CDI $(\rho=.51, p<.001)$, and PD and DC $(\rho=.56, p<.001)$, as well as within the SPPR: Satisfaction and Role Balance $(\rho=.53, p<.001)$. Moreover, we found statistically significant positive correlations within the PSQ: Social Exchange and Didactic $(\rho=.54, p<.001)$. The correlation between the PSI/SF and the SCL90-R are partially confirmed: PD and Depression $(\rho=.66, p<.001)$, and positive correlation was found between PD and Anxiety $(\rho=.55, p<.01)$. Also the negative correlations between the PSI/SF and the SPPR were confirmed in the maternal data: PD and Role Balance $(\rho=-.64, p<.001)$, TS and Role Balance $(\rho=-.58, p<.001)$. Interesting statistically significant negative correlations were also found between the SPPR and the SCL-90-R: Role Balance and Anxiety $(\rho=-.52, p=.008)$, Satisfaction and Hostility $(\rho=-.55, p=.004)$, Satisfaction and Phobic Anxiety $(\rho=-.55, p=.005)$, Satisfaction and Psychoticism $(\rho=-.54, p=.005)$.

Paternal data were interesting as well, where the positive correlations found on the whole dataset were confirmed within the PSI/SF: PD and P-CDI $(\rho=.54, p<.001)$, PD and DC $(\rho=.53, p<.001)$, P-CDI and DC $(\rho=.74, p<.001)$ and within the SPPR: Role Balance and Satisfaction $(\rho=.55, p<.001)$. The results are confirmed also for the correlations between the PSI/SF and the SCL-90-R: PD and Depression $(\rho=.58, p=.003)$, and PD and Hostility $(\rho=.64, p<.001)$, and additionally TS and Depression $(\rho=.55, p=.005)$, TS and Hostility $(\rho=.54, p=.006)$. Furthermore, negative correlations between the PSI/SF and the SPPR emerged as well: not only PD and Role Balance $(\rho=-.52, p=.001)$, and TS and Role Balance $(\rho=-.53, p<.001)$, but also P-CDI and Satisfaction $(\rho=-.53, p<.001)$, DC and Satisfaction $(\rho=-.52, p=.001)$, and TS and

Table 1

Mean scores (with Standard Deviation) and percentages of subjects in clinical range on the Parenting Stress Index-Short Form (PSI/SF) on 90 samples (47 mothers and 43 fathers). Percentages of subjects in the clinical range for each scale of PSI/SF were determined using normative guidelines provided in the PSI/SF Manual.

\begin{tabular}{|c|c|c|c|c|c|c|}
\hline & \multicolumn{3}{|l|}{ Mean $(S D)$} & \multicolumn{3}{|c|}{ \%Clinical range } \\
\hline & Mothers & Fathers & Total & Mothers & Fathers & Total \\
\hline PD & $55.0(27.3)$ & $54.2(26.6)$ & $54.6(26.8)$ & 26 & 23 & 24 \\
\hline DC & $81.6(20.7)$ & $79.5(23.6)$ & $80.6(22.0)$ & 72 & 63 & 68 \\
\hline P-CDI & 75.9 (20.6) & $77.7(21.3)$ & $76.7(20.8)$ & 47 & 53 & 50 \\
\hline TS & $74.9(21.8)$ & $74.2(24.2)$ & $74.6(22.8)$ & 49 & 51 & 50 \\
\hline
\end{tabular}

Notes: PD: Parental Distress, DC: Difficult Child Characteristics, P-CDI: Parent-Child Dysfunctional Interaction, TS: Total Stress. 
Table 2

Mean scores (with Standard Deviation) on the Parental Style Questionnaire and the Self-Perception of the Parental Role on 75 samples (39 mothers and 36 fathers).

\begin{tabular}{llll}
\hline & \multicolumn{2}{l}{ Mean $(\mathrm{SD})$} & Total \\
\cline { 2 - 4 } & Mother & Father & $3.94(0.47)$ \\
\hline Social Exchange & $4.11(0.44)$ & $3.76(0.44)$ & $3.39(0.47)$ \\
Didactic/Material & $3.48(0.54)$ & $3.29(0.36)$ & $4.10(0.62)$ \\
Limit Setting & $4.20(0.59)$ & $4.00(0.64)$ & $2.58(0.46)$ \\
Competence & $2.64(0.50)$ & $2.52(0.41)$ & $3.27(0.57)$ \\
Satisfaction & $3.37(0.48)$ & $3.16(0.64)$ & $2.41(0.33)$ \\
Investment & $2.44(0.34)$ & $2.37(0.32)$ & $2.79(0.53)$ \\
Role Balance & $2.82(0.55)$ & $2.76(0.51)$ & \\
\hline
\end{tabular}

Notes: Social Exchange, Didactic/Material and Limit Setting are the scales of the Parental Style Questionnaire. Competence, Satisfaction, Investment and Role Balance are the scales of the Self-Perception of the Parental Role.

Table 3

Mean scores (with Standard Deviation) on the Symptom Checklist-90-Revised on 52 samples (28 mothers and 24 fathers).

\begin{tabular}{llll}
\hline & Mean $(\mathrm{SD})$ & & Total \\
\cline { 2 - 4 } & Mother & Father & $0.46(0.40)$ \\
\hline SOM & $0.58(0.47)$ & $0.32(0.23)$ & $1.62(2.89)$ \\
O-C & $1.51(1.86)$ & $1.75(3.79)$ & $0.56(0.64)$ \\
I-S & $0.68(0.75)$ & $0.43(0.46)$ & $0.61(0.53)$ \\
DEP & $0.69(0.60)$ & $0.52(0.42)$ & $0.50(0.48)$ \\
ANX & $0.55(0.51)$ & $0.45(0.44)$ & $0.53(0.57)$ \\
HOS & $0.56(0.68)$ & $0.49(0.43)$ & $0.12(0.28)$ \\
PHOB & $0.13(0.34)$ & $0.11(0.21)$ & $0.59(0.61)$ \\
PAR & $0.70(0.69)$ & $0.47(0.50)$ & $0.26(0.40)$ \\
PSY & $0.30(0.49)$ & $0.21(0.25)$ & \\
\hline
\end{tabular}

Notes: SOM: Somatization, O-C: Obsessive-Compulsive, I-S: Interpersonal Sensitivity, DEP: Depression, ANX: Anxiety, HOS: Hostility, PHOB: Phobic Anxiety, PAR: Paranoid Ideation, PSY: Psychoticism.

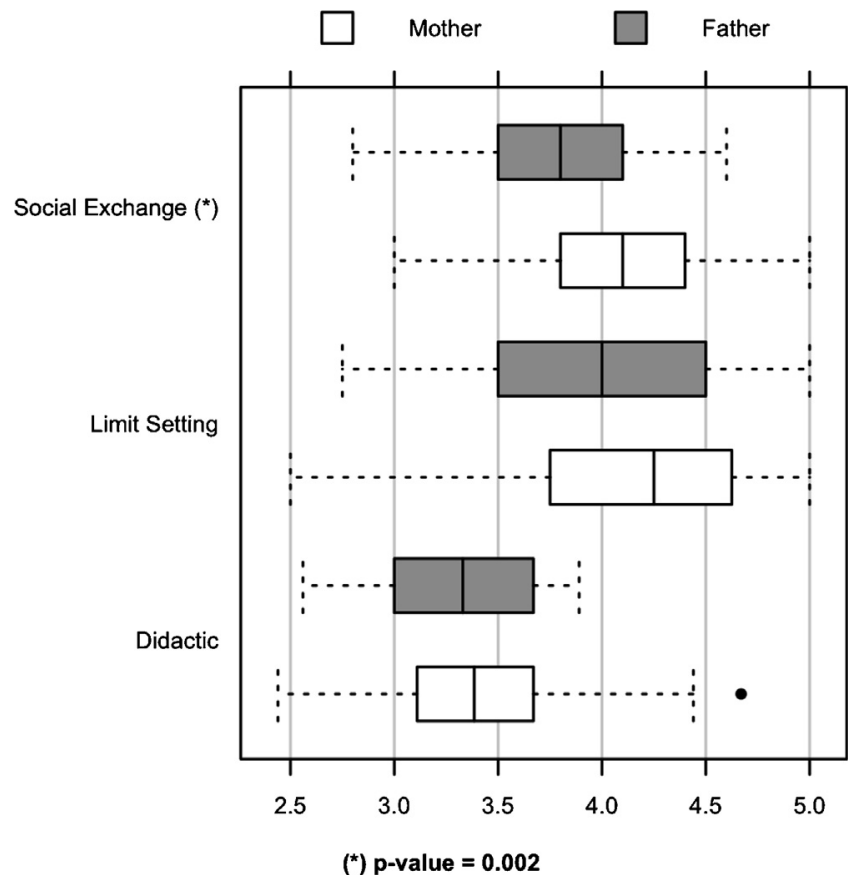

Fig. 1. Boxplots of the Parenting Style Questionnaire scores grouped by mothers (white) and fathers (gray). An asterisk highlights the scale having statistically significant difference by gender. 


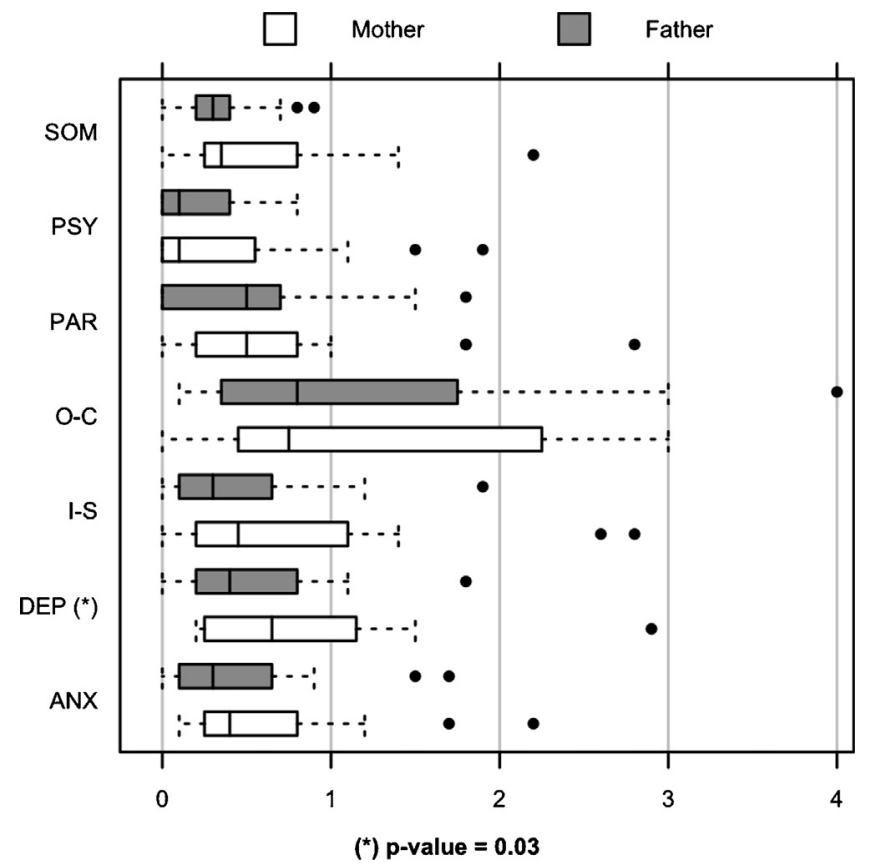

Fig. 2. Boxplots of the Symptom Checklist-90-Revised scores grouped by mothers (white) and fathers (gray). An asterisk highlights the scale having statistically significant difference by gender. SOM: Somatization, O-C: Obsessive-Compulsive, I-S: Interpersonal Sensitivity, DEP: Depression, ANX: Anxiety, HOS: Hostility, PHOB: Phobic Anxiety, PAR: Paranoid Ideation, PSY: Psychoticism.

Satisfaction $(\rho=-.58, p<.001)$. Interestingly, Limit Setting scale of the PSQ was correlated with several scales of the SCL-90R: Limit Setting and Interpersonal Sensitivity $(\rho=-.65, p=.001)$, Limit Setting and Anxiety $(\rho=-.57, p=.005)$, Limit Setting and Hostility $(\rho=-.52, p=.01)$, Limit Setting and Paranoid Ideation $(\rho=-.64, p=.001)$, Limit Setting and Psychoticism $(\rho=-.66, p<.001)$.

Multiple regression analysis was used to test if the PD score was significantly predicted by other parenting domain measures. The results indicated a regression model with three predictors explained $64 \%$ of the variance (adjusted $R^{2}=.64$, $F(3,43)=28.52, p<.001$; see Table 4 ) of the dataset on which it was built and predicts the PD score on the 10 -fold crossvalidation test sets with a good accuracy (adjusted $R^{2}=.56$, as computed by the DAAG R package). These three predictors are the Role Balance, the Depression and the P-CDI scores. Fig. 4 shows the comparison between the predicted values of PD versus the real value.

\section{Discussion}

The purpose of this study was to examine the profiles of both parents of children with ASD addressing three important domains of parenting. Specifically, we aimed to determine whether mothers and fathers differ in terms of parenting stress, parental attitude and mental health. The second aim of this work was to examine the relationship between the parental attitudes toward childrearing, the mental health conditions of both parents and their experiences of stress. To the best of our knowledge, this is the first attempt to cover parenting a child with ASD in much detail. Our results highlighted both similarities and differences between mothers and fathers of children with ASD and the existence of a link between parenting distress and other parenting domains. Below, we discuss the results with respect to each parenting domain, providing possible interpretations.

We found differences among parents in terms of their scores on measures of attitude and mental health. These results are partially consistent with our hypothesis. Considering the parental attitude, we found that mothers reported higher score on the Social Exchange scale of the Parental Style Questionnaire than fathers. Social exchanges are affective interpersonal dyadic interchanges (Barnard \& Solchany, 2002; Kaye, 1982) that include rocking, kissing, comforting, smiling, and playful face-to-face contact (Bornstein \& Cote, 2004). A possible explanation of this finding lies in degree to which parents engage with their children. Our data suggest that mothers of children with ASD actually engage in more social behavior with their children than fathers. This result may be interpreted in light of the literature on the mother-child interaction in typical development. In a previous study, indeed, Italian mothers reported that they interacted with their young children in social as well as didactic ways (Senese et al., 2012). Our findings expand this result, by extending to mothers of children with ASD and introducing a comparison with fathers. These results further support the idea that mothers and fathers interact with and care for children in different and oftentimes complementary ways (Barnard \& Solchany, 2002; Parke, 2002). Also parenting responsibilities, like social exchange, usually fall to mother (Bornstein \& Sawyer, 2008). Considering that social interactions 


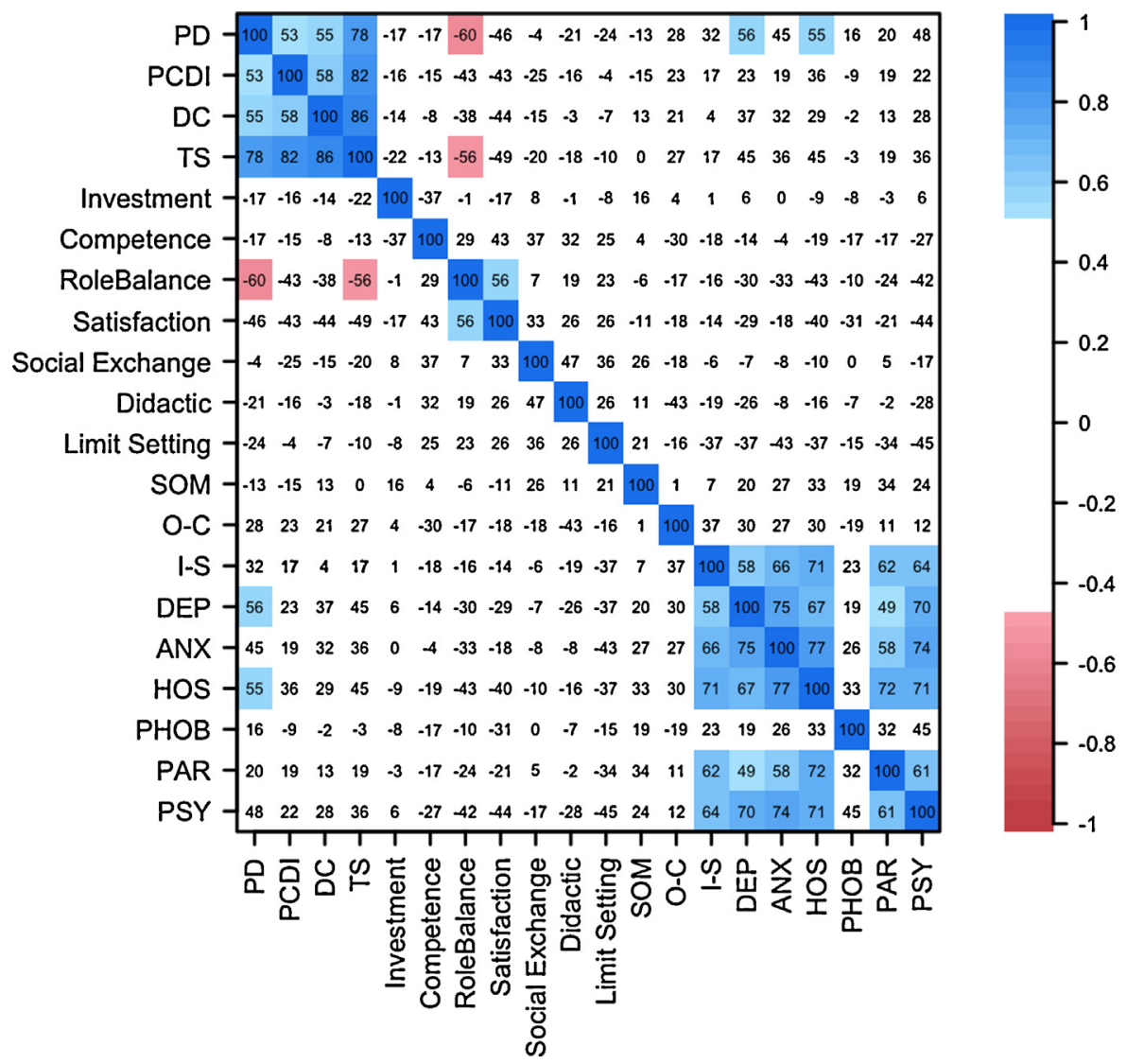

Fig. 3. Correlation plot on all parents' data. For improving readability, the correlation coefficients are multiplied by 100. Comparisons are color coded according to the association strength: blue indicating a strong positive correlation and red indicating a strong negative correlation. PD (Parenting Distress), P-CDI (Parent-Child Dysfunctional Interaction), DC (Difficult Child), and TS (Total Stress) are from the Parenting Stress Index-Short Form (PSI/SF). Investment, Competence, Role Balance, and Satisfaction are the scales of the Self-Perceptions of the Parental Role (SPPR). Social Exchange, Didactic, and Limit Setting are from the Parental Style Questionnaire (PSQ). SOM (Somatization), O-C (Obsessive-Compulsive), I-S (Interpersonal Sensitivity), DEP (Depression), ANX (Anxiety), HOS (Hostility), PHOB (Phobic Anxiety), PAR (Paranoid Ideation), and PSY (Psychoticism) are the Symptom Checklist-90Revised (SCL-90-R). (For interpretation of the references to color in this text, the reader is referred to the web version of the article.)

include different behaviors which parents use to engage children in visual, verbal, affective and physical interpersonal exchanges (Bornstein, 2002; Senese et al., 2012) and mothers tend to take on more parenting responsibilities (Bornstein \& Sawyer, 2008), it could be that, compared to fathers, mothers in our sample may take on more responsibility for the social interaction than fathers. In other words, parenting attitude of mothers and fathers differ in social behaviors to their children.

Table 4

Predictors of the Parental Distress (PD).

\begin{tabular}{lcr}
\hline & Model & \\
\cline { 2 - 3 } & Estimate & StdE \\
\hline (Intercept) & $59.3776^{* * *}$ & 19.3255 \\
Role balance & $18.9628^{* * *}$ & 5.0236 \\
P-CDI & $0.4385^{* * *}$ & 0.1126 \\
Depression & $21.8309^{* * * *}$ & 4.7527 \\
(Adjusted) $R^{2}$ & 0.6422 & \\
F & 28.52 & \\
BIC & 411 & \\
CV (Adjusted) $R^{2}$ & 0.5509 & \\
\hline
\end{tabular}

Note: $N=47$ (removed 43 samples with incomplete information). StdE = standard error $\mathrm{BIC}=$ Bayes Information Criterion, $\mathrm{CV}=$ cross-validation, P-CDI: Parent-Child Dysfunctional Interaction.

$$
\begin{aligned}
& { }^{*} p<0.01 . \\
& { }^{* *} p<0.001 . \\
& { }^{* * *} p<0.0001 .
\end{aligned}
$$




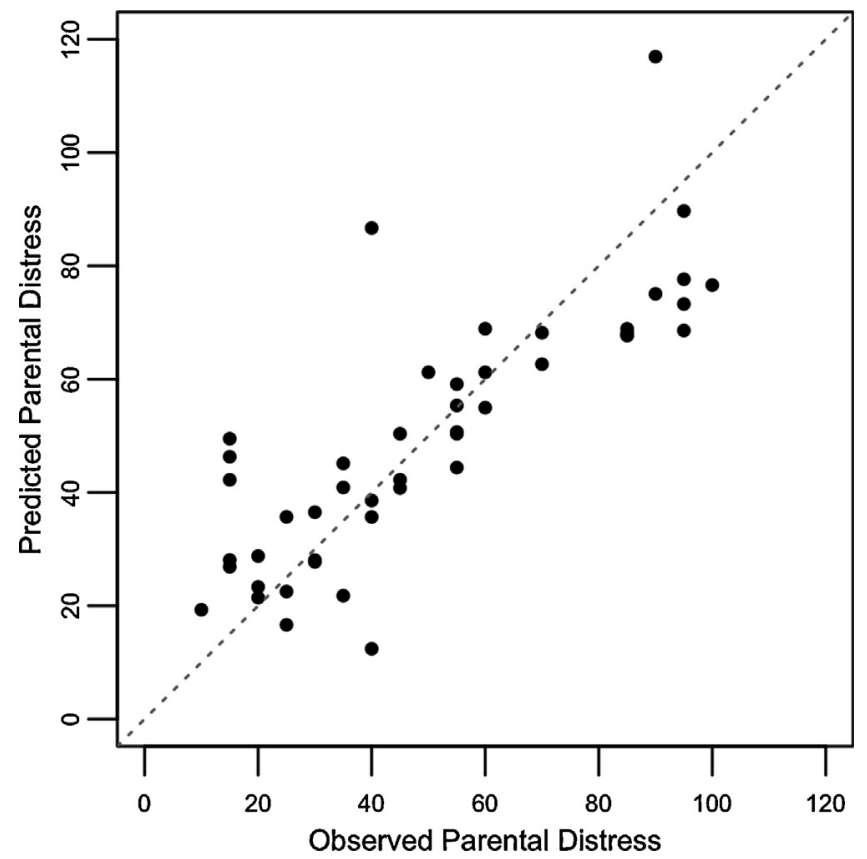

Fig. 4. Regression plot. The (observed) values of the Parental Distress (PD), on the $x$ axis, are compared with the values predicted by the three variables regression model, on the $y$ axis, which means by the Role Balance, the Depression and the P-CDI scores.

With respect to the parental mental health and well-being, in line with our hypotheses, a difference emerged from the Depression scale of the Symptom Checklist-90-Revised. This scale summarizes a wide range of accompanying symptoms of a depressive syndrome (i.e., withdrawal, loss of motivation, suicidal thinking, loss of vital energy; see Derogatis \& Lazarus, 1994). In our study, specifically, mothers of children with ASD reported higher level of depression than fathers of children with ASD. In the light of the present study and from the results of previous research, it appears as the difference among parents emerged in psychopathological problems, reflecting characteristics of the depressive syndrome. This result is consistent with those of Hastings and his colleagues (2005) who found gender differences in mental health with mothers of children with ASD reporting more problems than fathers, in terms of depression. Moreover, similar results have been found among parents of young newly diagnosed children (Davis \& Carter, 2008).

Why did mothers of children with ASD show higher level of depression than fathers? A possible explanation may concern the differences in responsibility assigned to parenting from each parent. As a matter of fact, historically, mothers remain the primary caregivers of their children in the vast majority of cultures around the world (Barnard \& Solchany, 2002), and they more likely assume the managerial role than fathers from their children's infancy through middle childhood (Parke, 2002; Russell \& Russell, 1987). Also, Winnicott (1965) described mothering as a "holding environment" for the child, and providing a holding environment requires that mothers have the physical and psychological resources to be on alert to the child (Barnard \& Solchany, 2002). Thus, parenting is often equated with mothering (Bornstein \& Sawyer, 2008). In line with these ideas, Olsson and Hwang (2001) suggested that mothers take on a larger part of extra care and practical work that the child with disabilities requires. In our sample, it could be that, mothers of children with ASD experience more depression symptoms compared to fathers of children with ASD, due to the fact that they are more involved in caregiving activities, they take more managerial responsibilities and therefore they are more exposed to the difficulties in rearing a child with special needs (Barnard \& Solchany, 2002; Crnic \& Low, 2002; Parke, 2002). However further studies are needed to confirm this hypothesis.

Besides those differences, we found also some similarities between mothers and fathers in terms of their scores on measures of parenting stress. The results show that there were no significant differences between mothers' and fathers' mean scores in all aspects of parenting stress (in all scales and total score of the Parenting Stress Index-Short Form). Although these results differ from some published studies (Moes et al., 1992; Sharpley et al., 1997; Tehee et al., 2009), they are consistent with those of other studies suggesting that mothers and fathers have similar parenting stress levels (Davis \& Carter, 2008; Hastings, 2003). Moreover, it is important to note that considerable percentages of mothers and fathers have scores above the clinical cut-points, showing that they experience clinically significant levels of parenting stress. Taken together, these results point out that both mothers and fathers of children with ASD have elevated parenting stress, consistent with the previous study of Davis and Carter (2008) which reported that many mothers and fathers of recently diagnosed toddlers share high levels of stress. The experience of raising a child with ASD can be stressful on individual parents, as both mothers and fathers of children with ASD have been shown to experience significant stress. Our results 
showed that the levels of parenting stress did not really differ between mothers and fathers of children with ASD, in line with others studies on mothers and fathers of typically developing children (Baker-Ericzn et al., 2005; Rao \& Beidel, 2009). Given the unique and important challenges in raising a child with ASD, these results highlight the need to consider paternal stress in addition to maternal stress in the further research and also in family interventions.

Other important findings from our study are the positive correlations found between the Parenting Distress and the Depression, the Parenting Distress and the Parent-Child Dysfunctional Interaction, and the negative correlation between the Parenting Distress and the Role Balance in all subjects. It is worth noting that, these are also the most consistent predictors of the Parenting Distress with respect to the results of our multiple regression analysis. This suggests that those parents who have high levels of depression symptoms, high levels of stress related to the interaction with their children with ASD, and less balance of the diverse roles in their life, are more likely to experience high stress in their role as a parent and personal factors that are directly related to parenting. In other words, the level of depressive symptom, the interaction system between them and their child, and the way in which parents balance their roles link to the degree of stress related parental functioning. These findings are consistent with those of Phetrasuwan and Shandor Miles (2009) that showed the relationship between high stress and more depression symptoms. Our results indicate that the psychological situations of parents, in particular depressive symptoms includes feeling hopelessness, lack of motivation, loss of vital energy is one of the sources of experiencing parenting distress. This finding highlights the importance of parents' own sense of well-being. Moreover, ineffectiveness in the integration of parents' diverse roles is linked to the parenting distress. A recent review study reports the impact of having a child with ASD extends beyond caregivers to the entire family (Karst \& Van Hecke, 2012). It is likely that parents are experiencing several challenges in balancing their role of employee, parent, spouse, caregiver, and this situation is another source of the parenting distress. In addition, stress as a function of dysfunctional interactions between parents and their child is related with the parenting distress. We believe that these three key aspects of parenting, namely parental attitude, parental mental health and stress related to the difficulties in parent-child interaction, are important to define and understand parenting stress in order to gain a deeper understanding of parenting a child with ASD.

Moreover, it is noteworthy that two scales of the Parenting Stress Index are correlated with a specific aspect of the Autism Diagnostic Observation Schedule-Generic (ADOS-G). The Communication scale of the ADOS-G is positively correlated with the Difficult Child score of fathers and with the Total Stress score of mothers. In other words, the way in which children use to communicate with others is associated with child related stress of fathers; whereas this children's communication functioning is related with total stress of mothers. Our results are partially in line with the previous study which showed the correlation between child behavior problems and mothers' stress (Hastings, 2003). Interestingly, in our sample, this association is emerged within fathers only from stress related to the characteristics of children that make them difficult to manage. On the other hand, within mothers, it is related with all aspects of stress (parenting distress, stress related to the interaction with their children, and child-related stress). Considering that mothers take more managerial responsibilities than fathers in caregiving activities (Parke, 2002; Russell \& Russell, 1987), it is likely that mothers and fathers may be affected differently by children's communication problems.

A further interesting result emerging from our analyses is the negative correlations for fathers between the Limit Setting scale of the Parenting Style Questionnaire and several scales of the Symptom Checklist-90-Revised (Interpersonal Sensitivity, Anxiety, Hostility, Paranoid Ideation and Psychoticism). Limit Setting is a scale in which parents reported themselves how frequently they actually engage in this specific parenting behavior, in this case it represents emphasizing rule keeping, firmness in disciplining and mannerliness in the child. This parenting style includes many ways in which parents guide children's behavior (Bornstein \& Cote, 2004). In our sample, the result showed that the Limit Setting is associated with the level of a broad range of symptoms for fathers. For mothers, instead, the Satisfaction dimension of the Self-Perceptions of the Parental Role is negatively correlated with a set of scales of the Symptom Checklist-90-Revised (Hostility, Phobic Anxiety and Psychoticism). A previous study of Furey and Forehand (1984) showed the relationship between maternal parenting satisfaction and depression, whereas, in our sample, the self-perception of parenting satisfaction is associated with the level of Hostility, Phobic Anxiety, Psychoticism symptoms. Taken together, mothers' and fathers' mental health conditions are associated with being satisfied and behaving such a limit setting. It indicates that the more mothers feel satisfied in their caregiving relationships, the less level of several psychological symptoms are reported and the more fathers think that they interact with their children in a limit-setting way, the less level of several psychological symptoms are expressed. These cognitions reflect important aspects about the experience of parenting and play a role in child development (Bornstein et al., 2003). The findings of the current study enhance our understanding of maternal and paternal attitude, showing the associations with diverse psychological conditions of mothers and fathers who have a child with ASD.

Finally, a number of important limitations need to be considered in the present work. First of all, although the sample size was enough to perform the correlations analysis for each gender, the multiple regression analyses could be done only within all subjects, not specifically within mothers or fathers; due to the smaller validation dataset within each gender to build the best linear model. With a larger sample sizes for both genders, it would be possible to examine the predictors of parenting stress for mothers and fathers separately. Therefore, further research is needed to concentrate on the investigation of the predictors of mothers and fathers. Moreover, although the measures applied in this work are widely used, they bring an issue in that the results emerge from self-reported data. More broadly, research is also needed to determine parenting domains using different methodological approaches in addition to the parents' self-reports.

In conclusion, the current study on families of children with ASD provides insights that will help researchers and clinicians to better understand the complexity of the treatment of children with ASD. Specifically, the results make 
noteworthy contributions to the current literature. First of all, we included fathers who are typically less considered in the literature, even though fathers are important as mothers in children's development (Parke, 2002). Second, we took into consideration several important domains of parenting: stress, attitude and mental health; whereas most studies have only been carried out with a small number of parenting domains (e.g., stress). Taken together, the current findings add to a growing body of literature on parenting and autism.

In addition, this study has clinical implications and parental practical importance. An implication of these findings is that both mothers and fathers should be taken into account but considering the differences and similarities between them in the parenting dimensions since these dimensions affect child development and have effects on parenting (Bornstein \& Lansford, 2010; Bornstein, 2002; Darling \& Steinberg, 1993; Foster et al., 2008; Jacob \& Johnson, 1997; Senese et al., 2012). Different parental style and well-being between mothers and fathers, which we found in the present study, bring diverse parental experience, and eventually practical effects on parent-child relation. Moreover, having higher level of stress in both parents is worth to consider in the clinical implications and parental practice. Therefore, in line with our findings, specific intervention programs are needed for both parents aimed to a reduction in their stress levels with taking into account the different parental style and well-being between mothers and fathers. Those interventions might benefit from a greater understanding of the relationship between stress and other parenting domains. Moreover, professionals might take into account a more effective father involvement in the children's life to make fathers to assist their children and also mothers. It may help to share the responsibilities in the life of their children, and decrease the experience of stress and depression. In this way professionals would be able to assist both parents comprehensively in becoming aware of important factors to focus and help them to cope efficiently with stress using suitable strategies.

\section{Acknowledgements}

We sincerely thank the families for their time and participation. The project was supported by the Observation, Diagnosis and Education Lab at the University of Trento, Italy.

\section{References}

Abidin, R. R. (1995). Parenting stress index: Professional manual (3rd ed.). Odessa, FL: Psychological Assessment Resources.

American Psychiatric Association (2013). DSM-V-TR ${ }^{\mathbb{R}}$ diagnostic and statistical manual of mental disorders. Arlington, VA: APA.

Baker, D. B. (1994). Parenting stress and ADHD: A comparison of mothers and fathers. Journal of Emotional and Behavioral Disorders, 2(1), 46-50.

Baker-Ericzn, M. J., Brookman-Frazee, L., \& Stahmer, A. (2005). Stress levels and adaptability in parents of toddlers with and without autism spectrum disorders. Research and Practice for Persons with Severe Disabilities, 30(4), 194-204.

Barnard, K. E., \& Solchany, J. E. (2002). Mothering. In M. H. Bornstein (Ed.), Handbook of parenting, Vol. 3: Being and becoming a parent (2nd ed., pp. 3-25). Mahwah, NJ: Lawrence Erlbaum Associates.

Beckman, P. J. (1991). Comparison of mothers' and fathers' perceptions of the effect of young children with and without disabilities. American Journal on Mental Retardation, 95(5), 585-595.

Bornstein, M. H. (2002). Parenting infants. In M. H. Bornstein (Ed.), Handbook of parenting, Vol 1: Children and parenting (2nd ed., pp. 3-43). Mahwah, NJ: Lawrence Erlbaum Associates.

Bornstein, M. H., \& Lansford, J. E. (2010). Parenting. In M. H. Bornstein (Ed.), Handbook of cultural developmental science (pp. 259-277). New York, NY: Taylor \& Francis Group.

Bornstein, M. H., \& Sawyer, J. (2008). Family systems. In K. McCartney \& D. Phillips (Eds.), Blackwell handbook of early childhood development (pp. 380-398). Oxford, UK: Blackwell Publishing Ltd.

Bornstein, M. H., \& Venuti, P. (2013). Genitorialità: fattori biologici e culturali dell'essere genitori. Bologna: Il Mulino.

Bornstein, M. H., Haynes, O. M., O’Reilly, A., \& Painter, K. (1996). Solitary and collaborative pretence play in early childhood: Sources of individual variation in the development of representational competence. Child Development, 67, 2910-2929.

Bornstein, M. H., Tamis LeMonda, C. S., Pascual, L., Haynes, O. M., Painter, K. M., Galperín, C. Z., et al. (1996). Ideas about parenting in Argentina, France, and the United States. International Journal of Behavioral Development, 19(2), 347-367.

Bornstein, M. H., Cote, L. R., \& Venuti, P. (2001). Parenting beliefs and behaviors in northern and southern groups of Italian mothers of young infants. Journal of Family Psychology, 15(4), 663-675.

Bornstein, M. H., Hendricks, C., Hahn, C. S., Haynes, O. M., Painter, K. M., \& Tamis LeMonda, C. S. (2003). Contributors to self-perceived competence, satisfaction, investment, and role balance in maternal parenting: A multivariate ecological analysis. Parenting, 3(4), $285-326$.

Bornstein, M. H., \& Cote, L. R. (2004). Mothers' parenting cognitions in cultures of origin, acculturating cultures, and cultures of destination. Child Development, 75(1), 221-235

Cohen, D. A., \& Rice, J. (1997). Parenting styles, adolescent substance use, and academic achievement. Journal of Drug Education, 27(2), 199-211.

Crnic, K., \& Low, C. (2002). Everyday stresses and parenting. In M. H. Bornstein (Ed.), Handbook of parenting, Vol. 5: Practical issues in parenting (2nd ed., pp. 243267). Mahwah, NJ: Lawrence Erlbaum Associates.

Darling, N., \& Steinberg, L. (1993). Parenting style as context: An integrative model. Psychological Bulletin, 113(3), 487-496.

Davis, N. O., \& Carter, A. S. (2008). Parenting stress in mothers and fathers of toddlers with autism spectrum disorders: Associations with child characteristics. Journal of Autism and Developmental Disorders, 38(7), 1278-1291.

De Falco, S., Esposito, G., Venuti, P., \& Bornstein, M. H. (2008). Fathers' play with their Down syndrome children. Journal of Intellectual Disability Research, 52(6), 490-502.

Deater-Deckard, K. (2004). Parenting stress. New Haven, CT: Yale University Press.

Derogatis, L. R., \& Lazarus, L. (1994). SCL-90-R, brief symptom inventory, and matching clinical rating scales. In M. Maruish (Ed.), The use of psychological testing for treatment planning and outcome assessment (pp. 217-248). Hillsdale, NJ: Lawrence Erlbaum Associates.

Eisenhower, A. S., Baker, B. L., \& Blacher, J. (2005). Preschool children with intellectual disability: Syndrome specificity, behaviour problems, and maternal wellbeing. Journal of Intellectual Disability Research, 49(Pt 9), 657-671.

Estes, A., Munson, J., Dawson, G., \& Koehler, E. (2009). Parenting stress and psychological functioning among mothers of preschool children with autism and developmental delay. Autism, 13(4), 375-387.

Fan, X., \& Chen, M. (2001). Parental involvement and students' academic achievement: A meta-analysis. Educational Psychology Review, 13(1), 1-22.

Flippin, M., \& Crais, E. R. (2011). The need for more effective father involvement in early autism intervention: A systematic review and recommendations. Journal of Early Intervention, 33(1), 24-50. 
Foster, C. J. E., Garber, J., \& Durlak, J. A. (2008). Current and past maternal depression, maternal interaction behaviors, and children's externalizing and internalizing symptoms. Journal of Abnormal Child Psychology, 36(4), 527-537.

Furey, W. M., \& Forehand, R. (1984). An examination of predictors of mothers' perceptions of satisfaction with their children. Journal of Social and Clinical Psychology, 2(3), 230-243.

Gau, S. S. F., Chou, M. C., Chiang, H. L., Lee, J. C., Wong, C. C., Chou, W. J., et al. (2012). Parental adjustment, marital relationship, and family function in families of children with autism. Research in Autism Spectrum Disorders, 6(1), 263-270.

Gottfried, A. W. (1985). Measures of socioeconomic status in child development research: Data and recommendations. Merrill-Palmer Quarterly, 31(1), 85-92.

Gottman, J. M., \& Wilson, B. J. (2002). Marital conflict, repair, and parenting. In M. H. Bornstein (Ed.), Handbook of parenting, Vol. 4: Social conditions and applied parenting (2nd ed., pp. 227-258). Mahwah, NJ: Lawrence Erlbaum Associates.

Guarino, A., Di Blasio, P., D’Alessio, M., Camisasca, E., \& Serantoni, G. (2007). PSI Parenting Stress Index-Forma breve-Per l'identificazione precoce di sistemi relazionali genitore-bambino stressanti. Firenze: Giunti OS.

Hastings, R. P. (2003). Child behaviour problems and partner mental health as correlates of stress in mothers and fathers of children with autism. Journal of Intellectual Disability Research, 47(Pt 4-5), 231-237.

Hastings, R. P., Kovshoff, H., Ward, N. J., Espinosa, F. D., Brown, T., \& Remington, B. (2005). Systems analysis of stress and positive perceptions in mothers and fathers of pre-school children with autism. Journal of Autism and Developmental Disorders, 35(5), 635-644.

Hoffman, C. D., Sweeney, D. P., Hodge, D., Lopez-Wagner, M. C., \& Looney, L. (2009). Parenting stress and closeness: Mothers of typically developing children and mothers of children with autism. Focus on Autism and Other Developmental Disabilities, 24(3), 178-187.

Hollingshead, A. A. (1975). Four-factor index of social status. New Haven, CT: Yale University unpublished manuscript.

Jacob, T., \& Johnson, S. L. (1997). Parent-child interaction among depressed fathers and mothers: Impact on child functioning. Journal of Family Psychology, 11(4), 391-409.

Karst, J. S., \& Van Hecke, A. V. (2012). Parent and family impact of autism spectrum disorders: A review and proposed model for intervention evaluation. Clinical Child and Family Psychology Review, 15(3), 247-277.

Kaye, K. (1982). The mental and social life of babiesbar. Brighton: Harvester Press.

Lord, C., Rutter, M., DiLavore, P. C., \& Risi, S. (2003). The autism diagnostic observation schedule (3rd ed.). Los Angeles, CA: Western Psychological Services.

MacPhee, D., \& Benson, J. B. (1986). Influences on maternal self-perceptions. Infant Behavior and Development, 9, 236.

McGrath, E. P., \& Repetti, R. L. (2000). Mothers' and fathers' attitudes toward their children's academic performance and children's perceptions of their academic competence. Journal of Youth and Adolescence, 29(6), 713-723.

Melson, G. F., Ladd, G. W., \& Hsu, H. C. (1993). Maternal support networks, maternal cognitions, and young children's social and cognitive development. Child Development, 64(5), 1401-1417

Moes, D., Koegel, R. L., Schreibman, L., \& Loos, L. M. (1992). Stress profiles for mothers and fathers of children with autism. Psychological Reports, 71(3f), 1272-1274.

Olsson, M. B., \& Hwang, C. P. (2001). Depression in mothers and fathers of children with intellectual disability. Journal of Intellectual Disability Research, 45(6), 535543

Parke, R. D. (2002). Fathers and families. In M. H. Bornstein (Ed.), Handbook of parenting, Vol. 3: Being and becoming a parent (2nd ed., pp. 27-73). Mahwah, NJ: Lawrence Erlbaum Associates.

Perry, A., Sarlo-McGarvey, N., \& Factor, D. C. (1992). Stress and family functioning in parents of girls with Rett syndrome. Journal of Autism and Developmental Disorders, 22(2), 235-248.

Phetrasuwan, S., \& Shandor Miles, M. (2009). Parenting stress in mothers of children with autism spectrum disorders. Journal for Specialists in Pediatric Nursing, 14(3), 157-165.

Rao, P. A., \& Beidel, D. C. (2009). The impact of children with high-functioning autism on parental stress, sibling adjustment, and family functioning. Behavior Modification, 33(4), 437-451.

Moura, M. L. S. d., Soares, I. D., Gomes, A. A. d. N., \& Bornstein, M. H. (2003). Socioeconomic status in Brazilian psychological research: I. Validity, measurement, and application. Estudos de Psicologia (Natal), 8(3), 375-383.

Rodrigue, J. R., Morgan, S. B., \& Geffken, G. R. (1992). Psychosocial adaptation of fathers of children with autism, Down syndrome, and normal development. Journal of Autism and Developmental Disorders, 22(2), 249-263.

Rossi, G. (1994). La rilevazione del SES in due contesti culturali italiani. AA. VV. Giornata di studio: "Lo stile dell'interazione madre-bambino in differenti contesti culturali italiani". Potenza: Università degli studi della Basilicata.

Russell, G., \& Russell, A. (1987). Mother-child and father-child relationships in middle childhood. Child Development, 58(6), $1573-1585$.

Sarno, I., Preti, E., Antonio, P., \& Madeddu, F. (2011). SCL-90-R Symptom Checklist-90-R Adattamento italiano. Firenze: Giunti OS Organizzazioni Speciali.

Schwarz, G. (1978). Estimating the dimension of a model. The Annals of Statistics, 6(2), 461-464.

Senese, V. P., Bornstein, M. H., Haynes, O. M., Rossi, G., \& Venuti, P. (2012). A cross-cultural comparison of mothers' beliefs about their parenting very young children. Infant Behavior and Development, 35(3), 479-488.

Seybold, J., Fritz, J., \& Macphee, D. (1991). Relation of social support to the self-perceptions of mothers with delayed children. Journal of Community Psychology, 19(1), 29-36.

Sharpley, C. F., Bitsika, V., \& Efremidis, B. (1997). Influence of gender, parental health, and perceived expertise of assistance upon stress, anxiety, and depression among parents of children with autism. Journal of Intellectual and Developmental Disability, 22(1), 19-28.

Tehee, E., Honan, R., \& Hevey, D. (2009). Factors contributing to stress in parents of individuals with autistic spectrum disorders. Journal of Applied Research in Intellectual Disabilities, 22(1), 34-42.

Theule, J., Wiener, J., Tannock, R., \& Jenkins, J. M. (2010). Parenting stress in families of children with ADHD: A meta-analysis. Journal of Emotional and Behavioral Disorders, 21(1), 3-17.

Venuti, P., \& Senese, V. P. (2007). Un questionario di autovalutazione degli stili parentali: uno studio su un campione italiano. Giornale italiano di psicologia, 34(3), 677-698.

Venuti, P., de Falco, S., Giusti, Z., \& Bornstein, M. H. (2008). Play and emotional availability in young children with Down syndrome. Infant Mental Health Journal, 29(2), 133-152

Winnicott, D. W. (1965). The maturational processes and the facilitating environment. London: Hogarth Press. 